\title{
Scaling Up Broken Systems? \\ Considerations from the Area of Music Streaming
}

\author{
Peter Knees
}

\begin{abstract}
We discuss the effects and characteristics of disruptive business models driven by technology, exemplified by the developments in music distribution and consumption over the last 20 years. Starting from a historical perspective, we offer insights into the current situation in music streaming, where technology has not only changed the way we access music but also has important implications on the broader ecosystem, which includes the consumers, the authors, the record industry, and the platforms themselves. The discussion points to potential benefits, as well as to the risks involved in the currently deployed systems. We conclude that the increased profitability of the disruptive business models in the music domain and beyond is largely generated at the expense of the providers of the goods or services being brokered. Using the platforms as a consumer further subsidizes their value and might lead to mono- and oligopolies. While technology allows companies to effectively scale up business, the resulting systems more often amplify existing injustices than mitigate them.
\end{abstract}

\section{Introduction}

Technological developments have always created opportunities for business. This is especially the case for disruptive technologies. In the past decades, we have increasingly seen a fascination of venture capital with businesses promising disruption in their area and therefore maximizing investment return. Some popular examples of this paradigm include platforms for retail (Amazon, Alibaba), accommodation (Airbnb, Booking.com), individual transportation (Uber, Lyft), and media distribution (Netflix, Spotify) or, e.g., fintech services (Klarna, TransferWise). Characteristic to these business models across all domains is some form of incentive, such as improved user experience and/or reduced cost, for the various stakeholders involved, and increased efficiency by automating central elements of the traditional business

\footnotetext{
P. Knees $(\bowtie)$

Vienna University of Technology, Vienna, Austria

e-mail: peter.knees@tuwien.ac.at 
approach, therefore allowing to scale up easily. These advantages undoubtedly are a key element for the success of the new business forms and overall the Internet-driven economy. Looking at the old-fashioned, obviously imperfect, and ultimately replaced business models reveals many flaws and inherent injustices. However, comparing them with their disruptive successors, it shows that the stakeholders consisting of a group of individual, non-organized participants (the providers of goods, as well as the consumers) tend to further lose power and influence in favor of the business owners providing the service. This raises the question: are these digital technologies merely permitting to scale up systems that were broken from the beginning? And if so: are disruptive digital technologies just amplifying injustices of the prior status quo?

In the following, we ask this question from the perspective of the area of music distribution. Music played an early and prominent role in the digital transformation of media delivery and consumption and has undergone significant changes over the last 25 years from a brick-and-mortar retail good to a ubiquitously available, de facto free service. We aim at giving a historical perspective describing the situation and the different stakeholders involved prior and past the disruption of the business and give an account on the beneficiaries and benefactors of this process, before drawing parallels to other areas of business disruption.

\section{A Brief History of Music Business}

Historically, the development of music production, distribution, and consumption is tightly intertwined with the technical possibilities of their respective time. Consider, for instance, the consequences of being able to transmit, physically record, and play back acoustic signals and the resulting means of broadcasting music and decoupling performance and listening. Over the second half of the twentieth century, this has led to the establishment of a record industry that would shift focus and power away from the composers of music and the publishing of sheet music toward the performers and marketing of events and trends. Revenue in this system was mostly generated by selling physical records on various types of media, most prominent on vinyl, music cassette, and compact disc (CD), with various stakeholders (among others, composers, performers, record labels, publishers, producers, distributors, and retailers) ideally being remunerated according to their negotiated share. Other, comparatively minor sources of revenue would stem from royalty payments based on airplay, performance, and licensing, as well as concerts. Radio was (and is) primarily a marketing tool to promote artists and records. To ensure payment to the various stakeholders, royalty collecting agencies established themselves to act on behalf of the rights owners, setting up rules for money distribution based on measurable, albeit ultimately arbitrary criteria, such as chart positions and other industry-conceived mechanisms. Essentially, this yielded a division of the area of music into "superstars" that could easily claim a (large, actually overproportioned) share and those who would have to fight for and often yield theirs. 
Overall, this system favored the record companies over the individual artists and creators, as controlling the music production means would allow them to exert power over artistic decisions and aggregate various licensing rights while maintaining strong ties to the media and other distribution channels. In practice, the market was controlled by the major record labels and a number of gatekeepers, establishing an oligopoly that works for a few at the core of the industry at the expense of the many contributors. As a side effect of this model, however, record companies would also afford to finance the development of new artists, i.e., to invest in artists and repertoire $(A \& R)$. For independent artists, the diversity of media outlets and distribution channels would nonetheless allow them to find niches and address potential audiences.

\section{The Disruption of the Business}

The end of the twentieth century brought new technological developments, again impacting the music industry - and again, before all others. The adoption of the Internet as communication medium and the invention of audio data compression algorithms and formats, specifically MP3, led to active exchange of digital music files among users via decentralized peer-to-peer networks such as Napster, outside all established channels, infrastructures, and monitoring mechanisms built by the music industry. Needless to say, the resulting, almost instant availability of comprehensive music repositories to every peer of the network posed a threat to the core business model of the industry. Instead of embracing the new technological opportunities, the developers and users of the networks were criminalized and the downfall of popular musicians and music as an artform itself foretold as the music industry would not be able to keep up the monetization strategy that supported all stakeholders, related businesses, and intermediaries involved. As a reaction, the entertainment industry successfully lobbied for changes in copyright and intellectual property rights laws to further strengthen their position.

A few years into these developments, which indeed brought declining profits for the music ecosystem in comparison to the peak of CD sales in 2001 (IFPI 2021), new players and services explored the potential of the informatization of music reproduction and the decoupling from a physical media in alternative business models. These ranged from traditional e-commerce models of digital stores for music files to newer paradigms such as flat-rate subscriptions or freemium models typically with an ad-based entry level for unlimited music streaming. Together with the rise of smartphones that pushed an "always online" philosophy, new forms of access to music were enabled.

Generally, this framework of digital distribution via online services offers many advantages over the historic approach and potential for mitigations to its shortcomings for the various stakeholders. For music consumers, instant, cheap, and legal access to large amounts of music becomes feasible. For music creators, including those who contribute to the production process, it can instantly provide traceable 
delivery of their music to their audiences without having to deal with the various gatekeepers and distribution hubs. For the industry in between, logistics of physical retail is no longer necessary. For the music platform itself, being the central hub for delivering music to users allows them to track music listening behavior and build personalized services such as recommender systems and advanced interfaces for music discovery (Knees et al. 2020). In this scenario, however, record labels might lose their position of power and be bypassed easily. Their bargaining chip to retain relevance in this development is the control over the back catalogues, i.e., licensing access to records by well-known and popular artists whose availability is imperative for broad adoption. Ultimately, the disruption of the business introduced streaming platforms as additional central market players, next to record companies, whereas brick-and-mortar retail has largely vanished.

\section{The Status Quo}

While sales of physical media have been declining since 2001, the rising revenue from streaming has almost compensated for the losses and delivered an overall turnaround in 2015, today constituting the largest source of revenue (IFPI 2021). With more than 440 million paid subscription users worldwide (ibid.), streaming is the dominating modality of (traceable) music consumption. Current catalogues of the market-leading streaming platforms such as Spotify, SiriusXM Pandora, Apple Music, Amazon Music, or YouTube Music offer up to 70 million music tracks (Spotify 2021). To sum up, music streaming has managed to scale up the music business both in terms of content accessible and users reached.

But how is this situation still (or even more?) profitable with more players to support? The payments made by streaming companies for individual streams are extremely small — on average about USD 0.004 per stream (see Pastukhov (2019)) — and often even negligible in sum per artist. Moreover, this amount is paid to the owners of the master recordings, which are typically the record labels, which then pass on only a small fraction to the artists. A reason for this practice is that revenue from streaming is often not explicitly negotiated in contracts, particularly in those signed before the technology existed. Hence, record companies benefit from licensing their back catalogues as it allows them to keep the largest share of the royalty payments to themselves. Also, recent efforts to present more justified and "fair" money distributions models, e.g., by distributing subscribers' fees according to their individual listening preferences, as implemented in Deezer's user-centric payment system (Deezer 2021), are not improving the situation as again the money is not paid directly to the artists, but the producers and publishers. Furthermore, despite the technical possibility to trace all plays of individual tracks, information about play counts across various platforms remains opaque for music creators, especially on platforms driven by user-generated content like YouTube on which content identification focuses again on the needs of big rights owners and neglects independent artists. These shortcomings themselves led again to the establishment of disruptive 
businesses like Kobalt or UnitedMasters that combine the roles of independent labels, rights management, and publishing companies, providing easier access to platforms and increased transparency of the process in a unified interface.

Record companies can now focus their attention and marketing strategies to a few distribution channels instead of dealing with a variety of different outlets. Artists and repertoire $(A \& R)$ is not part of their core business anymore, as new trends and talents are observed on social media platforms and co-opted based on hypes. As streaming is not generating much revenue, alternative sources of income like live performances, merchandise, sponsoring, and licensing of tracks need to provide a stable financial foundation for artists. At the same time, record labels increasingly demand a share of this profit in exchange for managing and promoting artists.

While record labels have once more found their position at the center of the business at the expense of artists and music creators, the question of profitability of the streaming platforms remains. Based on the annual reports of the various platforms, it appears they are not (yet?) operating profitable businesses. Their value seems to lie elsewhere, namely, in the potential for commercially exploiting their user basis, not only by charging for the service, but in fact by providing a data basis for marketing analyses sold to brands and advertising (Zuboff 2018). This might explain why, for instance, Spotify is partly owned by an intricate structure of the biggest record companies and rights holders, consisting of Universal Music Group, Warner Music Group, Sony Music, and, most recent, Tencent (Ingham 2020; see also Eriksson et al. 2019).

\section{$5 \quad$... And Beyond?}

While in the music business the old industry reinvented their role and managed to ultimately shape the new music industry based on its assets and absorb the disruptive element, in other domains, disruption has led to a replacement of the intermediaries and gatekeepers. In the movie domain, Netflix, first as a recommender-driven mail-in service, then as a movie streaming pioneer, completely eliminated the established video and DVD rental business and opened up a market that is now a battleground for several competitors following the same model. In the transportation area, Uber entered a long-time undisputed market and took large shares by storm. In the tourism domain, platforms like Booking and Expedia pushed the traditional business model of travel agencies close to the edge of the cliff (while the COVID-19 crisis seemingly finally pushed it over).

While Web-based services are at the center of all these examples and provide a new dimension of user experience in all these domains, it is never the user side that makes the business profitable. In music, the changed industry landscape is carried by besting the composers and creators of music due to old contracts or reduced benefits. In transportation, the pressure is put on drivers to make up for reduced wages by increasing customer throughput. In the tourism domain, hotels are put under further pressure to reduce their own profit margins and undersell their capacities to get 
visibility on platforms. In the movie domain, on the other hand, the competition of streaming services and their newly founded studios seems to increase the opportunities within the movie and TV industry rather than forcing the producers and workers to produce more for less compensation. In this context, it should be noted that the movie industry, especially in the USA, is strongly unionized.

The user is not spared from possible negative consequences either. Resorting again to the music example, the status quo of having access to virtually all music all the time is a utopian situation for music aficionados, and the technical developments that have enabled this situation over the last decades are a shining example of the blessings of information technology. At the same time, users become strongly dependent on and locked into such platforms. On one hand, "having access" does not mean "to own," and "personal collections" consisting of playlists created on platforms might become incomplete or missing due to changed circumstances in licensing. On the other hand, such services become more indispensable the more they are used. This might lead to the more general observation that "the rise in market concentration is greater in industries that are more intensive users of digital technologies" (Qureshi 2019); i.e., the disruption of industries by means of digital technologies itself promotes the emergence of mono- or oligopolies. In the long run, this is disadvantageous for the user and most other stakeholders involved.

To conclude, technology drives and "sells" disruptive business models. The increased profit of these models is often and largely generated by exploiting the people who provide the actual value behind the product, especially if they are not organized to fight for their joint interests. The users further subsidize these businesses simply through usage. From the perspective of Digital Humanism, this situation is unsatisfactory. We should first use technology to overcome the many broken systems before helping scaling them up.

\section{References}

Deezer (2021). Deezer wants artists to be paid fairly. Online: https://www.deezer.com/ucps, retrieved: 05/02/2021.

Eriksson, M., Fleischer, R., Johansson, A., Snickars, P., \& Vonderau, P. (2019). Spotify Teardown: Inside the Black Box of Streaming Music. Cambridge: MIT Press.

IFPI (2021). Global Music Report 2021. Online: https://gmr2021.ifpi.org/assets/GMR2021_State\% 20of\%20the\%20Industry.pdf, retrieved: 05/02/2021.

Ingham, T. (2020). Who Really Owns Spotify?. Rolling Stone. Online: https://www.rollingstone. com/pro/news/who-really-owns-spotify-955388/, retrieved: 05/02/2021.

Knees, P., Schedl, M., \& Goto, M. (2020). Intelligent User Interfaces for Music Discovery. Transactions of the International Society for Music Information Retrieval, 3(1), pp.165-179. DOI: https://doi.org/10.5334/tismir.60 
Pastukhov, D. (2019). What Music Streaming Services Pay Per Stream (And Why It Actually Doesn't Matter). Soundcharts. Online: https://soundcharts.com/blog/music-streaming-ratespayouts, retrieved: 05/02/2021.

Qureshi, Z. (2019). Inequality in the Digital Era. Work in the Age of Data. BBVA OpenMind Collection (12), BBVA.

Spotify (2021). Company Info. Online: https://newsroom.spotify.com/company-info/, retrieved: $05 / 02 / 2021$.

Zuboff, S. (2018). The Age of Surveillance Capitalism: The Fight for the Future at the New Frontier of Power. London: Profile Books.

Open Access This chapter is licensed under the terms of the Creative Commons Attribution 4.0 International License (http://creativecommons.org/licenses/by/4.0/), which permits use, sharing, adaptation, distribution and reproduction in any medium or format, as long as you give appropriate credit to the original author(s) and the source, provide a link to the Creative Commons license and indicate if changes were made.

The images or other third party material in this chapter are included in the chapter's Creative Commons license, unless indicated otherwise in a credit line to the material. If material is not included in the chapter's Creative Commons license and your intended use is not permitted by statutory regulation or exceeds the permitted use, you will need to obtain permission directly from the copyright holder. 